\title{
Enseigner la sortie de cadre pour changer les habitudes
}

Marion M. Bisiaux, Laetitia Fontaine et Nicole Rege Colet

\section{(2) OpenEdition \\ Journals}

Édition électronique

URL : http://journals.openedition.org/ripes/2037

ISSN : 2076-8427

Éditeur

Association internationale de pédagogie universitaire

Référence électronique

Marion M. Bisiaux, Laetitia Fontaine et Nicole Rege Colet, « Enseigner la sortie de cadre pour changer les habitudes », Revue internationale de pédagogie de l'enseignement supérieur [En ligne], 35(1) | 2019, mis en ligne le 11 avril 2020, consulté le 08 septembre 2020. URL : http://journals.openedition.org/ ripes/2037

Ce document a été généré automatiquement le 8 septembre 2020

Article L.111-1 du Code de la propriété intellectuelle. 


\title{
Enseigner la sortie de cadre pour changer les habitudes
}

\author{
Marion M. Bisiaux, Laetitia Fontaine et Nicole Rege Colet
}

\section{Osons sortir du cadre!}

1 Toute innovation comprend sa part de risque, et il en est de même pour l'innovation pédagogique. C'est toujours une aventure hors des sentiers battus, sans but préétabli, ni outil de navigation, une expérience faite de découvertes pour un (ré-)enchantement possible du monde. C'est aussi une expérience renversante qui bouscule à la fois nos manières de penser la pédagogie et nos pratiques pédagogiques. Puis, avec le temps et les premiers résultats, vient l'envie de partager les observations et découvertes. Après la sortie du cadre, sciemment voulue, sera-t-il possible d'y revenir pour raconter notre parcours? Nous faisons le pari que les lecteurs accueilleront avec intérêt notre récit d'expérience.

2 L'atelier matières à construire, ci-après amàco, est résolument tourné vers l'innovation et l'exploration de l'apprentissage de soi et du monde en s'appuyant sur une philosophie singulière, celle des matières à construire. Cet article présente une de ses expériences pédagogiques, un module de formation autour des matériaux innovants pour la construction durable, conçu et dispensé en 2015 et 2016 à l'école d'ingénieurs de l'Institut national des sciences appliquées de Lyon (ci-après Insa Lyon). Terrain d'expérimentation pédagogique de la philosophie d'amàco, ce module permet d'observer les effets sur les apprentissages des étudiants ainsi que la transformation des pratiques pédagogiques des enseignants. Ce récit d'expérience est aussi l'occasion d'un premier bilan, après six années de fonctionnement, dans le cadre d'un mandat d'innovation pédagogique.

Notre récit commence par expliciter notre démarche hors cadre et les balises qui ont guidé nos explorations. Nous présentons ensuite les fondements de la philosophie d'amàco et la manière dont les principes sont intégrés dans l'approche pédagogique. Nous évoquons ce qui nous incite à innover et à imaginer une pédagogie qui bouscule 
les habitudes ainsi que la manière dont nous nous sommes mis à l'écoute des changements émergents dans un paysage en mutation. Trois aspects en émergence sont discutés dans les parties consacrées à nos observations, à savoir l'interdisciplinarité, les interdépendances entre tradition et innovation et la créativité. Nous concluons avec une réflexion quant à l'effet des interventions d'amàco pour transformer les pratiques pédagogiques des enseignants.

\section{Une démarche innovante pour explorer l'innovation pédagogique}

4 Le récit d'expérience est un genre qui cherche encore sa place dans un environnement qui privilégie la " démarche recherche » centrée sur un questionnement a priori. Dans le cas de cette dernière, les chercheurs se mettent en quête de réponses provisoires ou d'éléments d'approfondissement; le questionnement guide les choix méthodologiques ainsi que l'interprétation des données récoltées. Il détermine ensuite la manière de rendre compte de la démarche et le genre scriptural.

5 Dans la «démarche innovation" utilisée ici, les protagonistes se distancient des repères usuels; ils se lancent dans l'aventure en se mettant directement à l'écoute à l'écoute de ce qui est en transformation dans un environnement décrit comme volatile, incertain, complexe et ambigu et qui, ce faisant, échappe à toute lecture à travers les prismes traditionnels de la recherche en sciences de l'éducation. L'innovation se déroule en temps réel et de manière continue. Les observateurs privilégiés s'efforcent de capturer cette expérience au fil du temps et d'y donner sens sans nécessairement recourir à des conceptualisations préétablies ou à des outillages conventionnels.

6 Par essence, notre démarche d'investigation autour du module de formation innovant que nous présentons dans cet article s'inscrit dans une approche exploratoire. Nous entrons dans la démarche en nous efforçant de poser un regard curieux sur nos données, sans hypothèse préconçue. Cela nous conduit à pouvoir dégager ce qui se situe en arrière-fond, sous le bruit de surface, et qui correspond à un nouveau paysage pédagogique en devenir. Nous verrons plus loin comment cette démarche s'aligne sur les valeurs fondamentales d'une philosophie de la matière en amont de l'expérimentation pédagogique.

7 Nos principales sources d'inspiration pour entamer cette exploration sont le "Scholarship of Teaching and Learning», la théorie ancrée (grounded theory), les théories du changement émergent, théories qui accompagnent de nombreuses démarches d'innovation pédagogique. Notre récit d'expérience trouve sa forme dans un genre assez distancié des structures habituelles d'articles scientifiques. Les contextualisations y sont volontairement raccourcies, mais soutenues par des références à nos sources d'inspiration, tandis que nous avons choisi de réserver l'espace à l'expression des personnes impliquées.

\section{3. À l'origine, une philosophie de la matière}

8 À l'origine de l'atelier amàco se trouve une philosophie des matières à construire développée à partir de multiples sources d'inspiration dont celle portée par le laboratoire CRAterre, pionnier de la valorisation de la construction en terre en France 
et dans le monde. Cette philosophie part du constat que la plupart des bâtiments construits, aujourd'hui, révèlent une déconnexion entre l'humain et son milieu (Anger et Fontaine, 2016). À l'inverse, certaines architectures vernaculaires, construites avec les ressources environnementales et sociales de leurs territoires, véhiculent des émotions qui invitent à une reconnexion entre la matière dont nous sommes tous faits et celle qui constitue le monde. Aujourd'hui, le déséquilibre écologique nous fait prendre conscience de l'urgence de retisser ces liens.

Amàco a choisi de s'intéresser plus spécifiquement à la restauration des relations avec les matières brutes et peu transformées, telles que la terre, le bois, la paille, utilisées pour les besoins de la construction et nécessitant peu d'énergie pour être mises en œuvre. Amàco propose de réapprendre à s'émerveiller devant ces matières ordinaires, souvent déconsidérées au profit d'une illusion de la modernité, puis de prendre conscience des forces invisibles qu'elles contiennent et de leurs interactions avec nousmêmes. Pour que cette prise de conscience puisse être à l'origine de changements concrets dans les attitudes et les comportements, amàco travaille à fournir à ses interlocuteurs les moyens de sortir des sentiers battus, afin qu'ils puissent être en mesure d'imaginer et d'utiliser ces matériaux qui ne dominent pas le marché ou dont les filières n'existent pas encore. S'agissant, ici, d'un changement de cadre de référence au sens de Mezirow (1997), les formations imaginées nécessitent plus qu'une démarche purement cognitive qui, souvent, peut inhiber l'intuition procurée par les émotions et les sensations (Damasio, 1995) : elles visent une prise de confiance en soi, en l'autre et, surtout, en la matière.

\section{Amàco, une possibilité d'expérimenter}

10 Depuis 2012, amàco a pu trouver un terrain d'expérimentation de sa philosophie de la matière et de ses intentions pédagogiques dans le cadre d'un financement Idefi Initiative d'excellence en formation innovante - issu du Programme des investissements d'avenir du gouvernement français ${ }^{1}$, qui vise, entre autres, à promouvoir l'innovation. Ce financement, qui s'achève à la fin 2019, apporte les moyens pour déployer des dispositifs pédagogiques sur le long terme et en assurer le suivi. Dans le cadre de ce financement, quatre établissements d'enseignement supérieur se sont réunis pour fonder amàco. Ce partenariat a permis de développer des politiques pédagogiques avec les équipes de direction pédagogiques concernées contribuant, entre autres, à essaimer au sein des établissements le renouveau pédagogique. Fin 2017, amàco s'est constitué en association dont les membres du conseil d'administration sont les représentants des établissements fondateurs.

$11 \mathrm{Au}$ croisement des cultures scientifiques, techniques, artistiques et architecturales, amàco a pour vocation d'être un centre de formation et d'expérimentation autour de la matière. Les activités de formation et de diffusion des connaissances sont menées conjointement avec des activités de recherche appliquée et d'accompagnement d'opérations réelles dans le domaine de la construction, qui font appel à des matières premières disponibles sur les territoires. L'offre de formation s'adresse à différents publics touchant à la fois les étudiants en écoles d'architecture et en écoles d'ingénieurs, les professionnels du monde de la construction et le grand public.

12 Aujourd'hui, amàco comprend une équipe de 14 salariés réunissant les compétences de l'architecte, de l'artisan, de l'ingénieur matière et matériaux, de l'ingénieur 
pédagogique, de communication, etc. Cette équipe est accompagnée de prestataires de services qui interviennent notamment dans les domaines de la production artistique, des techniques de mise en œuvre, de l'artisanat ou encore de la pédagogie. Amàco dispose enfin de marraines et de parrains qui apportent leur soutien et leur expertise au projet.

Dans le domaine de la construction, la notion du durable (ou de construction durable) est difficile à aborder tant elle est chargée de valeurs, souvent politisées en raison de l'idée que l'écologie devient désormais un droit commun. L'ingénieur, appelé à respecter un idéal d'impartialité, veillera à ne pas compromettre sa légitimité en adoptant des pratiques pouvant être qualifiées de militantes. À l'inverse, la notion d'innovation bénéficie d'une image positive et parait plus accessible. Combiner durable et innovation représente un rapprochement acceptable pour l'ingénieur qui cherche à concilier sa pratique professionnelle avec les enjeux environnementaux.

L'innovation pédagogique portée par amàco se situe assez loin de l'injonction à l'innovation utilitariste. Dans un monde où l'individu est déraciné de son substrat, elle vise à reconstruire des liens porteurs de sens entre l'individu et les matières à construire (Lemaître, 2015). L'enjeu consiste à fournir à l'apprenant des conditions pour qu'il prenne conscience, à travers son corps, son cœur et son esprit, de l'existence de solutions viables et réalistes pour construire mieux et de manière durable. La démarche pédagogique favorise le dialogue avec la matière qui devient une source de créativité et d'inventivité.

Notre approche pédagogique consiste à apprendre la matière par la matière, à travers l'expérimentation. Les formations s'articulent autour de trois axes. Le premier propose de découvrir les surprenantes propriétés physiques, mécaniques, chimiques, esthétiques, artistiques ou techniques des matières brutes et peu transformées utilisées dans la construction. Cette étape vise à bousculer les préconceptions au sujet de ces matières. Le deuxième axe de formation encourage à prendre conscience de l'importance des sens, du corps et des émotions dans nos relations avec les matériaux d'origine naturelle, puis d'étendre cette prise de conscience aux liens qui existent entre la matière, l'individu et la société (Bisiaux, Tric, Houben, Fontaine, Anger et Berthiaume, 2017). Enfin, le troisième axe consiste en l'expérimentation de ces matières dans des situations concrètes afin d'amorcer une transformation profonde des conceptions autour de la construction.

16 Amàco donne la part-belle à l'intelligence du corps, valorisant la pratique, tout en encourageant la liberté d'expérimentation plutôt que la répétition de gestes normés. Ainsi, les ateliers de mise en pratique de techniques de construction sont associés à des phases d'expérimentation de la matière. Des exercices sensoriels, au cours desquels les étudiants découvrent les matières ou les matériaux, les yeux bandés, sont proposés pendant les ateliers ou les cours théoriques. Ces phases de pratique sont également complétées par l'utilisation de démonstrations en direct, ou de vidéos présentant des professionnels en train de faire, ou de mettre la main à la pâte.

17 Amàco apporte également des enseignements théoriques tant dans les ateliers que dans les cours magistraux. Ils prennent la forme de conférences expérimentales, de vidéos et de visuels soignés. Ils ont pour spécificité d'aborder le sujet de la matière de façon interdisciplinaire. Ils se basent pour cela sur la science des matériaux, sur des exemples d'architecture vernaculaire et contemporaine, sur l'esthétisme de la matière, sur des 
expériences contre-intuitives, le tout dans le but de susciter la curiosité et l'émerveillement, puis de semer le désir d'apprendre.

18 L'art permet de dépasser les limites de l'expérimentation et de s'affranchir des besoins rationnels. $\mathrm{Au}$ cours d'ateliers intensifs, des séances de créativité puis d'expérimentation libre sont proposées à partir d'une bibliothèque de matières. Dans les cours magistraux, les étudiants découvrent des œuvres d'artistes travaillant la matière, identifient et partagent leurs ressentis à leur propos.

Enfin, amàco estime que la prise de conscience - et aussi de confiance - en ces nouvelles approches de la construction nécessite de s'appuyer sur des illustrations concrètes, par exemple, l'étude de travaux d'architectes contemporains utilisant les matières brutes comme matériaux, le partage de retours d'expérience sur les chantiers auxquels amàco et ses collaborateurs participent et, lorsque c'est possible, par la mise en œuvre à l'échelle réelle.

20 La construction durable fait également appel à des aspects sociétaux et de vivre ensemble. Être capable de travailler avec des approches interdisciplinaires et d'apporter sa propre contribution disciplinaire sont des compétences clés. C'est pourquoi, au cours des ateliers ou des cours théoriques, les étudiants travaillent ensemble pour faire émerger des solutions co-construites. Les formateurs se positionnent alors en accompagnateurs, encourageant les explorations autour des erreurs qui deviennent alors une réelle source d'apprentissage (Astolfi, 2015).

\section{Une pédagogie qui bouscule les habitudes}

21 Dans le domaine de la formation initiale, amàco collabore avec une vingtaine d'établissements d'enseignement supérieur en France et une quinzaine à l'étranger. De 2012 à 2017, il a contribué à la formation de près de 11000 apprenants. Toutes les formations sont conçues sur mesure avec l'enseignant ou l'équipe pédagogique responsable de l'enseignement en fonction des objectifs de formation. Elles peuvent prendre la forme d'une intervention de quelques heures ou de quelques jours, s'intégrer ponctuellement au cours d'un module ou d'un cursus de formation, ou encore occuper la totalité d'une unité d'enseignement.

Comme mentionné précédemment, amàco propose des contenus qui mêlent science de la matière, architecture, technique de construction, art, artisanat. Les dispositifs d'enseignement sont des conférences expérimentales ou participatives, des tables rondes, des ateliers encadrés ou créatifs, des ateliers sensoriels et plus encore. Les connaissances pédagogiques théoriques et pratiques sont partagées avec les enseignants partenaires et la plupart des contenus et méthodes sont rendus disponibles, pour une réutilisation libre et créative au-delà du contexte de formation.

\subsection{Plus qu'une collaboration, un partenariat placé sous le signe de la co-création}

23 L'Insa Lyon fait partie des établissements d'enseignement supérieur avec qui amàco a pu développer une collaboration privilégiée sur le long terme. Les deux initiateurs du projet amàco y ont mené leurs études et y ont développé des recherches et des expériences pédagogiques sur les sciences de la matière en grains. Objet d'une thèse de 
doctorat (Anger, 2011), ces travaux ont donné naissance, par exemple, au programme de recherche, de formation et de sensibilisation sur la construction en terre crue, nommé « Grains de Bâtisseurs ».

L'équipe d'amàco connaît donc bien l'enseignement et la recherche de l'Insa Lyon. De même, les enseignants du domaine des matériaux connaissent bien la philosophie d'amàco qu'ils ont vu naître. Les collaborations spontanées ont été le prétexte, en 2014, du test d'un dispositif pédagogique optionnel et hors cursus pour les étudiants de troisième, de quatrième et de cinquième année, avec deux enseignants de l'établissement. Ce dispositif de huit heures, intitulé « La terre crue comme matériau de construction durable », comprenait une conférence expérimentale, une conférence participative et un atelier pratique. Au total, 26 étudiants se sont inscrits et les questionnaires administrés à l'issue de la formation ont relevé un fort taux de satisfaction, satisfaction partagée par les enseignants de l'Insa et l'équipe d'amàco. L'expérience a été réitérée en 2015 et 2016, cette fois-ci intégrée au sein du cursus de l'Insa de cinquième année, dans le cadre d'une « option transversale » de 96 heures et intitulée "Matériaux Innovants pour la Construction Durable»(ci-après Micodu). Compte tenu de l'ampleur et de la durée de cette expérimentation, l'équipe d'amàco a souhaité documenter et observer les effets de ce module sur l'expérience formative des étudiants.

\subsection{L'option Micodu}

Les options transversales de l'Insa Lyon sont des formations inscrites au programme de cinquième année. Situées aux interfaces des disciplines, elles ont été créées afin de " préparer les élèves à la résolution de problèmes de complexité croissante au sein des métiers d'ingénieurs » (Fontaine, Moevius, Bisiaux, Neuville et Prud'homme, 2017, p. 1). Bien que nommées options, elles ont un caractère obligatoire puisque les étudiants doivent indiquer cinq options dans une liste de vœux, dont une seule leur sera attribuée.

Amàco et les enseignants partenaires des départements Génie civil et urbanisme (GCU) et Sciences et génie des matériaux (SGM) ont conçu l'option Micodu au croisement de plusieurs départements. Pour les enseignants impliqués, cette option était également " un moyen de permettre à des élèves-ingénieurs de plus en plus en quête de sens, d'horizontalité et d'engagement au service de la société, de se former à des technologies alternatives et de se construire un projet professionnel plus cohérent avec leurs aspirations et leurs valeurs » (Fontaine et al., 2017, p. 3). En effet, à l'époque, le sujet de la construction durable était très peu abordé dans les enseignements : par exemple, les matériaux de construction durable n'occupaient que 10 minutes d'un cours de 16 heures sur les matériaux. De plus, les méthodes d'enseignement utilisées pour enseigner les matériaux de construction durable étaient de type dit «transmissif». L'expérimentation Micodu a été conçue pour renouveler ces enseignements de l'Insa Lyon en intégrant les contenus et les méthodes pédagogiques développés par amàco.

Amàco a choisi d'expérimenter des dispositifs pédagogiques jamais utilisés pour l'enseignement des matériaux à l'Insa Lyon, tout en s'appuyant sur les contenus de cours préexistants des enseignants impliqués. Afin d'en assurer la crédibilité, une attention particulière a été apportée à l'ingénierie pédagogique en étroite collaboration 
avec les enseignants : formulation des objectifs d'apprentissage, sélection des contenus, des méthodes pédagogiques et des méthodes d'évaluation (Daele et Berthiaume, 2013). Les objectifs d'apprentissage portaient sur 1) la prise de conscience des enjeux de la construction durable, de la diversité des matériaux bruts ou peu transformés et leurs utilisations possibles, 2) la mise en œuvre d'une démarche d'exploration créative (démarche expérimentale, analyse scientifique), 3) le travail en équipe et une démarche proactive afin de s'insérer professionnellement dans le domaine des filières des matériaux bio et géo-sourcés.

L'option d'une durée totale de 96 heures comprenait 42 heures de face-à-face (cours, voyage d'études, conférences expérimentales), 4 heures de tables rondes et 50 heures de projet. Comme pour toutes les formations amàco, le module reposait essentiellement sur la manipulation des matières et l'expérimentation. À cela s'ajoutaient de multiples prises de contact avec des spécialistes et professionnels locaux dans le domaine des matériaux pour la construction. Au total, ce sont une quinzaine de formateurs qui sont intervenus dans le module: trois formateurs amàco, cinq enseignants-chercheurs de l'Insa et des intervenants extérieurs provenant d'autres établissements de formation, des architectes et des ingénieurs en bureau d'études.

Des moments d'évaluation informelle des apprentissages (jeux de questions-réponses, période de réflexion collective) ont été organisés tout au long de l'enseignement. Au terme de l'enseignement, une évaluation formelle a été effectuée associant, d'une part, un jury composé des enseignants de l'Insa et, d'autre part, les étudiants. Pour la notation, les pondérations suivantes ont été utilisées : $60 \%$ de la note issue de la note du jury; 20 \% issue d'une auto-évaluation par les étudiants du groupe évalué; $20 \%$ issue d'une note attribuée par chacun des étudiants lors des soutenances des groupes. Ce système d'évaluation s'inscrit dans une approche évaluative visant à renforcer les apprentissages plutôt qu'à les sanctionner (Rege Colet et Berthiaume, 2013). À la fin du module, un questionnaire de satisfaction a été distribué et un bilan de retour d'expérience a été organisé avec les enseignants impliqués.

\section{Une démarche d'investigation aux sources multiples pour défricher le paysage}

30 La démarche adoptée pour observer les effets novateurs de Micodu en temps réel, se focalise sur les apprentissages réalisés par les étudiants au terme de la formation et sur la transformation des pratiques d'enseignement des enseignants impliqués dans l'expérimentation. Les données rassemblées à cet effet croisent plusieurs sources d'information par une triangulation des propos des étudiants, ceux des enseignants de l'Insa Lyon et les observations des formateurs d'amàco.

31 Les données sont issues de méthodes mixtes combinant de l'observation participante, des entretiens individuels et des questionnaires. Le corpus de données comprend :

- Trois questionnaires d'évaluation de l'enseignement par les étudiants; il s'agit des questionnaires conçus pour les besoins du contrôle qualité interne. Ils ont été administrés en direct, à l'issue soit du dispositif test (2014), soit des options Micodu (2015 et 2016);

- Un questionnaire post-formation, envoyé en mai 2017 par courrier électronique aux 70 élèves ayant participé au dispositif test et aux options Micodu; 
- Un questionnaire post-formation «témoin", envoyé en juillet 2017 par courrier électronique à 350 anciens élèves n'ayant suivi ni le dispositif test, ni Micodu, mais diplômés de l'Insa Lyon dans les mêmes départements et les mêmes années;

- Quatre entretiens individuels avec des enseignants de l'Insa Lyon et d'amàco impliqués dans la conception et/ou la mise en œuvre de Micodu. a priori, leur degré d'assimilation. Nous étions également à l'écoute des tensions structurantes qui apparaissent inévitablement quand nous nous engageons dans une démarche transformationnelle où nous acceptons de changer nos pratiques. Qu'en estil pour les enseignants appelés à innover dans leurs pratiques d'enseignement? Comment les étudiants vivent-ils ces changements et les intègrent-ils dans leurs pratiques professionnelles? C'est une exploration par questionnement itératif, où 
chaque question abordée mène à d'autres et permet d'entrer plus profondément en contact avec les données pour entendre les forces invisibles qu'elles hébergent.

Qu'avons-nous découvert lors de nos explorations de l'expérience vécue? Les prochaines sections développent les lignes qui évoquent les transformations annoncées dans le paysage de l'enseignement de l'architecture et de la construction durable.

\section{L'interdisciplinarité pour rompre avec le cloisonnement disciplinaire} fin de formation montrent leur intérêt pour le mélange des départements et les méthodes qui favorisent le travail en équipe interdisciplinaire. Les commentaires soulignent l'originalité du mélange de cultures, par exemple: «Projet très intéressant principalement à cause du fait qu'il regroupe plusieurs départements. On a pu voir les différents parcours et l'influence des cours sur nos connaissances. J'aurais bien aimé pouvoir faire plusieurs projets inter-départements au cours de l'université. »

40 " [J’ai aimé] le mélange des élèves qui viennent de différents départements [...]. Il faudrait essayer de promouvoir l'option transversale auprès de davantage de départements que GCU/ SGM/GEN puisqu'elle est abordable et intéressante ».

41 "J'ai beaucoup appris, par les cours donnés mais aussi par l'expérimentation et les autres étudiants. [...] La transdisciplinarité est la clé, il faut réfléchir le tout. »

D'autres commentaires mentionnent les bénéfices de multiplier les références à travers la diversité des intervenants offrant, ce faisant, autant de regards et de points de vue variés.

43 Il n'en demeure pas moins que, dans les questionnaires post-formation, aucun répondant ne cite explicitement l'inter- ou la transdisciplinarité comme étant l'un des principaux apprentissages réalisés à l'issue de l'option transversale.

44 L'enquête post-formation montre que la quasi-totalité les étudiants se trouvent à l'aise dans le travail en équipe et apprécie de travailler dans une perspective interdisciplinaire. Il est à relever que les étudiants du groupe témoin ne semblent pas moins à l'aise que les étudiants Micodu, suggérant qu'il n'y a vraisemblablement pas de perception différente quant aux compétences acquises en matière d'interdisciplinarité en fonction des enseignements reçus. Une nuance interprétative s'impose : qu'ils aient suivi le dispositif innovant ou pas, les étudiants commencent tout juste à être exposés à une approche pédagogique construite autour d'objectifs d'apprentissage. Il y a sans doute encore du chemin à faire avant de pouvoir se prononcer, de manière consciente, sur les apprentissages réalisés et d'en rendre compte dans un questionnaire.

Du côté des formateurs, les enseignants considèrent que l'initiation au travail interdisciplinaire a été l'apport le plus important de Micodu, indépendamment de leurs préconceptions.

"Au début, j'étais un peu dubitatif: je me disais, ok pour les [étudiants de] GCU, SGM mais pas sûr que ça intéresse les autres. Et puis quand ça a commencé, on voyait que les horizons des 
étudiants étaient très différents, alors que pourtant ça ne faisait que trois ans qu'ils avaient quitté le tronc commun. Ceux qui venaient d'Informatique avaient carrément un autre discours, un autre langage. [...] Les étudiants de GCU et SGM devaient expliquer à leurs collègues d'informatique des choses qu'ils ne pensaient pas avoir besoin d'expliquer et se rendaient compte que ce n'était pas si facile. Sur mon ressenti, [...] je me souviens avoir été surpris de l'efficacité avec laquelle ils ont trouvé des solutions au problème. C'était assez créatif comme mélange de culture... Le plus important c'est vraiment les mélanges de culture, [...] ce qui est tout aussi important dans un métier d'ingénieur. » (E3)

Un enseignant reconnaît avoir surtout privilégié, au début, les objectifs d'apprentissage portant sur les aspects qualitatifs et techniques des nouveaux matériaux durables, bien que l'interdisciplinarité fasse également partie de ses objectifs. Sa participation effective au module lui a permis d'accorder plus d'attention aux effets de l'interdisciplinarité vécue: "L'aspect le plus intéressant paradoxalement, ce n'était pas directement la thématique, mais [...] c'était d'avoir vu travailler ensemble sur des projets, des gens qui avaient au départ des profils tous différents. [...] Ça m'a permis de me rendre compte que bien qu'ils soient tous des ingénieurs Insa, qui avaient presque tous fait le même parcours pendant deux ans, ils avaient des cultures complètement différentes. Et en plus, je suis convaincu que c'est à la fin du cursus [...], où ils ont été marqués, qu'ils ont les connaissances de la discipline, que c'est là où c'est le plus intéressant. »(E1)

L'intérêt de l'option Micodu en matière d'interdisciplinarité réside dans le fait qu'elle favorise l'intuition. La nouvelle manière d'aborder la construction en terre ou en fibres végétales forge un contexte contre-culturel qui permet "d'apporter des thématiques différentes, [...] des thématiques scientifiques, non-scientifiques [...], un peu en dehors des thématiques qu'ils travaillent habituellement » (E2).

L'accompagnement des étudiants sur les différentes thématiques est possible grâce à la variété des enseignants et des intervenants, une démarche à encourager pour autant qu'elle ne déséquilibre pas les emplois du temps des enseignants, "surchargés " par leur volume d'enseignement et leurs tâches administratives. Il s'agit de «mobiliser les gens pour faire quelque chose de nouveau, qui va demander un investissement, et bien ça devient dur. Il faut trouver le dernier recruté » (E2).

Les points de vue des étudiants et des enseignants révèlent des orientations différentes quant à la pertinence de l'interdisciplinarité. Tandis que les enseignants l'ont vécu comme une expérience transformant leur pratique professionnelle, les étudiants ne mentionnent pas explicitement d'apprentissage en matière d'interdisciplinarité. Dans le cas des enseignants, l'expérience révèle la réalité de l'environnement disciplinaire dans lequel leurs enseignements sont nichés. Ils observent, cependant, que les étudiants sont plus à l'aise que ce qu'ils auraient imaginé. Pour des diplômés, bien que marqués par cette expérience de décloisonnement disciplinaire en tant qu'élève de l'Insa Lyon, les distinctions entre départements se sont probablement atténuées dès l'entrée dans la vie professionnelle. En dehors de l'école, l'inter- et la transdisciplinarité deviennent une norme et des pratiques courantes. Pour eux, il n'y a pas nécessité d'établir des liens avec les apprentissages visés dans le cadre de Micodu. L'effet de causalité directe s'estompe en faveur d'un effet indirect. 


\section{La tradition et l'innovation : entre tiraillement et engouement}

51 Trois thèmes rendent compte de situations et de pratiques où apparaissent des tiraillements entre le besoin de respecter les traditions et l'envie d'innover. Plus particulièrement, les propos des enseignants évoquent des mouvements subtiles annonciateurs de transformations dans les domaines : 1) des contenus d'enseignement, 2) des méthodes d'enseignement et 3) de leur posture d'enseignant.

\subsection{Une innovation de contenu, place au durable}

52 Ce sont les apprentissages en lien avec la construction durable qui retiennent l'attention des étudiants. Dans les questionnaires de satisfaction, la plupart estiment avoir changé de regard sur la construction durable et avoir « envie de travailler avec des matériaux biosourcés ». Ceci est confirmé dans les questionnaires post-formation où une plus grande proportion d'étudiants estime prêter attention au caractère durable d'un projet de construction. Les commentaires font état d'une prise de conscience sur la possibilité de construire autrement et plus respectueusement de l'environnement, notamment le fait que les matériaux biosourcés puissent répondre aux besoins de la construction contemporaine. Une différence significative ressort entre les étudiants Micodu et notre groupe témoin dans un énoncé portant sur le recours aux matériaux bruts et peu transformés : les étudiants Micodu reconnaissent quasiment tous (98\%) qu'ils peuvent répondre aux enjeux de la construction contemporaine, contre seulement $78 \%$ du groupe témoin.

53 Pour de nombreux diplômés, les prises de conscience sont à l'origine de certaines de leurs pratiques actuelles, notamment leur positionnement par rapport à la construction durable.

54 «L'ensemble des connaissances théoriques et débats a contribué à façonner ma réflexion sur la construction, m'aidant dans mon travail actuel. »

55 «Je travaille dans une équipe d'études technique et de prix en maitrise d'ouvrage. Il est donc utile d'avoir des notions de développement durable pour avoir toujours plus d'arguments et de solutions pertinentes afin de formuler des propositions de projet de logement. »

56 Force est de constater une faible prise en compte du durable dans l'offre de formation de l'Insa Lyon. Un enseignant illustre cela pour son cours matériaux initialement tourné vers le béton, l'acier, le bois et les polymères et dont il a pris la charge à son arrivée dans l'établissement: "Quand j'ai repris les cours de matériaux, [...] je me suis aperçue que la partie que les étudiants avaient vraiment bien aimé, c'était la partie que je leur avais rajoutée [...] sur les éco-matériaux et la valorisation de l'argile. [...] J'avais trouvé qu'ils étaient très réceptifs et qu'ils étaient contents de voir autre chose que du béton et de l'acier. [...] Parce qu'en Génie civil et Urbanisme, ils étudient pendant trois ans du béton. On ne leur présente pas d'alternative en fait. Ça veut dire qu'on forme des ingénieurs en génie civil qui ne savent pas qu'on peut mettre autre chose que du béton ou de l'acier. [...] Cette année, dans la refonte du programme, sur les 16 heures de matériaux, j'ai décidé d'en prendre deux pour leur parler du matériau terre, des fibres, de ce qu'on pouvait faire avec, et de leurs propriétés. » (E2)

57 Les étudiants sont attirés par les thèmes innovants, ceux qui favorisent un éveil, une prise de conscience, ceux qui invitent à questionner les pratiques et les habitudes et à 
adopter de nouvelles approches plus alignées avec les besoins environnementaux et sociétaux. L'appel au changement et à la construction durable et respectueuse est entendu. Nous verrons plus loin comment cette attraction pour l'innovation peut être retenue par la force des habitudes.

\subsection{Une innovation de méthodes, la manipulation de la matière}

Pour les enseignants, la manipulation de la matière a été l'apport le plus important du module. Ils relèvent que l'introduction de l'expérimentation et des manipulations pendant les cours bousculent les traditionnelles méthodes transmissives d'enseignement. L'expérience a permis de prendre du recul sur la manière d'enseigner et d'en tester d'autres. Ces méthodes expérimentées et mises à l'épreuve sont ensuite intégrées dans d'autres enseignements, assurant de ce fait une transposition de l'expérimentation pédagogique vers d'autres enseignements.

"Cela m'a permis de me reposer des questions sur des phénomènes physiques que j'explique [aux étudiants], et ce que je peux leur montrer pour qu'ils comprennent le phénomène, sans avoir besoin d'avoir toutes les équations etc. [...] J'ai rajouté des vidéos et puis j'ai recentré sur l'essentiel. Et je le vois sur les partiels, je trouve que quand ils ont vu des vidéos de phénomènes, ils l'intègrent mieux [...], sans forcément avoir le bon vocabulaire, mais ils ont compris. Alors que sur les premières années où je n'avais des fois que des équations, je m'apercevais qu'ils avaient compris l'équation, mais qu'ils n'avaient pas compris ce que ça voulait dire. Ça c'est ce qui a changé avec Micodu et avec amàco » (E2).

La transposition conduit à introduire des manipulations scientifiques dans les cours magistraux sur les matériaux, mais également «sur d'autres enseignements, qui n'ont rien à voir avec la terre... ça marche » (E1). La volonté de dégager du temps pour davantage de manipulations est retenue. L'expérimentation reste un outil qui permettrait de «faire baisser la barrière d'énergie » (E3) pour aller vers une formalisation plus approfondie.

61 "La manipe, l'expérience, les réveille. Ils ont envie de comprendre. Puis, on leur donne les moyens de comprendre. Ce n'est pas sûr que ça influe sur l'examen, mais je pense que dans leur tête ça reste [...]. On leur a donné les moyens d'être assez malins pour aller chercher l'information et trouver la solution. Ça agit comme un marqueur de mémoire. Ils se disent : "Je ne me souviens plus de la loi, mais je sais que je peux trouver la solution quelque-part "” " (E3).

L'intérêt de la manipulation porterait moins sur la compréhension des théories, mais plutôt sur "une autre approche, beaucoup plus empirique, beaucoup plus expérimentale " (E4). Cette approche, qualifiée de compréhension intuitive de la matière (Bisiaux, Fontaine, Anger et Houben, 2015), semble indispensable pour adapter les apprentissages universitaires aux besoins des mondes professionnels. "C'est quelquechose qui m'a manqué pendant mes stages. Tout de suite j'ai voulu être extrêmement précis, alors qu'il aurait fallu que je dégrossisse le travail en étant très expérimental » (E4). Or, un module ne suffit pas à ce que les étudiants puissent vraiment bousculer leur vision de la matière et l'importance de la phase expérimentatrice : "Il faudrait qu'il y ait cet aspect empirique un peu dans chaque cours pour que ça rentre aussi dans leur manière de penser " (E4).

63 Pour les étudiants, le caractère innovant se situe non seulement dans la manipulation de la matière, mais plus généralement dans le format jugé original et efficace. Selon leurs commentaires dans les questionnaires de satisfaction, les méthodes favoriseraient leur engagement; ils qualifient les méthodes d'«intéressantes", "instructives », " 
enrichissantes » où encore permettant "d'accrocher et de s'investir plus que dans une option uniquement théorique ». Le plaisir d'apprendre transparaît également dans leurs propos : "Une ouverture sur la construction durable réussie et agréable », " Un projet qui donne envie de s'investir ", "J'ai apprécié le projet dans lequel il est facile de se plonger ».

Les réponses au questionnaire post-formation évoquent un lien entre l'expérience de méthodes pédagogiques originales et les apprentissages réalisés. Sont notamment cités "la complémentarité entre l'apprentissage théorique et pratique qui permet de s'approprier totalement des concepts", la possibilité "d'apprendre par une pédagogie différente que celle habituellement utilisée dans le cursus universitaire ", "l'approche par l'expérience et le sensible pour connaitre les propriétés et potentiels [du matériau terre]».

\subsection{Une innovation de posture enseignant, un guide qui valorise l'autonomie}

65 Un autre objectif important de l'option Micodu porte sur l'autonomisation des étudiants, notamment à travers leur participation active à la réalisation d'un projet d'équipe. Les enseignants observent que cela modifie sensiblement la relation entre enseignants et étudiants, où l'enseignant devient un guide et accorde plus de liberté aux apprenants (Rege Colet, 2016).

"Réfléchir avec [les élèves] change le fonctionnement du professeur, [...] on aborde les mêmes questionnements de thermique ou de formulation de matériaux [...] mais ils ne nous perçoivent pas pareil, on casse un peu la structure. Et c'est chouette. » (E2).

67 "C'était très différent de ce que l'on faisait d'habitude, [...] c'est l'aspect purement créativité, où l'on pouvait faire un peu ce qu'on voulait ». (E1)

68 Ces changements de posture sont également perçus par les étudiants qui le soulignent dans les questionnaires de satisfaction: «Très bonne approche des enseignants envers les étudiants, disponibles, qui nous considèrent comme de futurs ingénieurs. »

« Un bon dosage au niveau de l'encadrement [...], de bon conseil, mais nous laisse de l'autonomie et l'opportunité d'être créatifs. "

70 Ainsi, l'expérimentation et une démarche centrée sur l'autonomie des étudiants bousculent les relations pédagogiques; les relations hiérarchiques s'estompent en faveur de collaborations, où chacun, enseignant comme étudiant, devient une personne ressource et où l'accompagnement mutuel dans les explorations prime sur la transmission des contenus.

\section{Les fissures d'où peut surgir l'élan créatif}

71 Qu'est-ce qui pourrait freiner l'enthousiasme des étudiants comme celui des enseignants? La rencontre avec les exigences des milieux professionnels de la construction et leurs traditions! Les propos recueillis montrent que l'élan de créativité et l'envie d'explorer hors des sentiers battus se heurtent aux besoins ressentis d'être conformes aux attentes sociales pour assurer son insertion professionnelle.

Les propos des diplômés indiquent que leur motivation à s'orienter vers les matériaux de construction durable est entravée par des facteurs externes à leur champ d'action. 
Le premier concerne la faible représentation des matériaux non conventionnels dans l'offre de formation et dans les canaux de sensibilisation grand public.

"J'ai pu constater que les matériaux naturels, aussi complexes du point de vue scientifique et intéressants au niveau technique que les matériaux industriels [...], étaient négligés dans ma formation d'ingénieur. Au point de n'être même pas cités dans les différents cours que j'ai pu avoir dans ma spécialité "matériaux" ».

74 "Venant de GCU, je me rends compte que nous avons une vision tronquée des problématiques en se restreignant aux seuls bétons et aciers ".

75 Le second concerne la structuration actuelle du principal marché de la construction. Ce dernier est décrit comme verrouillé par un cadre, des normes, des lobbies et des enjeux économiques rendant difficile le passage à l'action et la structuration de filières alternatives.

76 "Avec la mise en place de normes toujours plus strictes en termes de performance et de sureté, le gros avantage de ces matériaux [...] est qu'ils sont bien connus avec une formulation maitrisée qui assure une répétabilité des processus et des résultats. Avec la terre, durable et locale, on est forcément dépendant du matériau local justement, donc cela demande un travail de RetD à chaque nouveau projet, et un investissement en termes de responsabilité de la part des entreprises. Je ne sais pas si elles sont prêtes à ce type de prise de risque ".

77 "Je retiens de ce cours que les matériaux durables ont prouvé, depuis longtemps, leur durabilité et bienfaits pour l'environnement, l'économie locale, la santé, la culture etc. [...] Pourtant ils ne font toujours pas le poids en face des gros lobbys de la construction, surtout le béton. Je me rappelle d'une intervention par un professionnel qui expliquait que pour imposer la terre comme matériau de construction, il fallait faire passer par et valider toute une série de règlements, de lois pour la sécurité, etc. Les barrières contre l'innovation sont immenses, c'est ça que je retiens de ce module! ».

78 Il n'en demeure pas moins que les diplômés ayant suivi l'option Micodu expriment plus leur envie de travailler avec des matériaux répondant aux enjeux du développement durable que les diplômés du groupe témoin. La « comparaison» des résultats avec le groupe témoin montre que les diplômés ayant suivi l'option estiment plus être en mesure de prendre en compte les enjeux du développement durable dans leurs pratiques personnelles et professionnelles sans que cela affecte leur employabilité. En effet, il n'y a pas de différence entre les deux groupes quant à la capacité perçue de trouver un emploi qui corresponde à leurs souhaits professionnels.

Bien que l'intérêt pour les matériaux biosourcés soit manifeste, le contexte actuel du monde de la construction - peu propice aux matériaux non-conventionnels - ne semble pas agir comme un frein. Cette posture pourrait refléter le choix d'être pragmatique, plutôt que militant. Malgré une formation très cadrée, durant laquelle les étudiants ont surtout abordé les matériaux normés, répondant principalement aux besoins des grandes filières de matériaux industrialisés (qui, par ailleurs, parrainent de nombreuses offres de stages), ces jeunes ingénieurs n'évoquent pas le risque d'être marginalisés, ni d'être considérés comme non-conformes à l'image que le grand public se fait de l'ingénieur. Au contraire, ils restent résolument positifs.

Que se passe-t-il du côté des enseignants? L'optimisme des étudiants se retrouve dans un document rédigé par un groupe d'enseignants-chercheurs en sciences humaines et sociales de l'Insa Lyon au titre évocateur de Ingénieur.e.s engagé.e.s (Fontaine et al., 2017; Neuville, Bouyer et Lefèvre, 2017). Ce document évoque la présence d'étudiants 
brillants, motivés et impliqués dans des questions sociétales mais, paradoxalement, moins engagés dans leurs études d'ingénieurs. Pour y parer, le groupe «ingénieur.e.s engagé.e.s» suggère de revoir l'offre pédagogique en favorisant les démarches expérimentales qui permettrait d'acquérir, puis de mobiliser les compétences clés de l'ingénieur de manière fluide et congruente. Le groupe a choisi l'expérience Micodu comme modèle.

81 Cet appel a trouvé une forte résonnance auprès des enseignants Micodu qui discutent autant les questions d'engagement que de militantisme.

"L'idée [d'ingénieur.e engagé.e.s] ce n'est pas forcément d'avoir des étudiants qui soient militants. C'est vraiment de leur donner une ouverture scientifique et l'ouverture pour aller vers ce vers quoi ils veulent aller. [...] C'est au-delà des étudiants qui ne se retrouvent plus, c'est aussi pour les enseignants qui ne se retrouvent plus dans leur boulot. » (E2)

"Je pense qu'il faut faire attention, car il y a différentes formes d'engagement, [...] il y a des gens qui peuvent être très bien dans le système et fortement engagés dans le système. Il faut être prudent, je ne veux pas faire de jugement de valeurs. [...] La démarche est intéressante car ça peut répondre à une attente de certains élèves [...]. Il faut juste faire attention dans le discours. Je me méfie toujours du militantisme. » (E1)

Restent la force des habitudes et le poids des mentalités sociales qui forgent les organisations et les pratiques. Pour les enseignants, un premier frein se situe très clairement au niveau de leur emploi du temps et de la solitude pédagogique des pionniers. «En fait, je n'aimerais faire que ça, si je pouvais, si on pouvait m'aider. C'est trop d'énergie pour le faire tout seul. [...]. J'ai une barrière d'énergie qui m'empêche d'aller plus loin. » (E3). L'enseignant E2 mentionne également « le sentiment d'être seule, la première année, où on avait trouvé personne" comme limitant son investissement. Plus impliqué, il considère que sa participation à l'option a justement été l'occasion de s'appuyer sur ses collègues : "J'ai bien apprécié de travailler sur la mise en place [de l'option], réfléchir à plusieurs. Ça parait bête, mais finalement dans nos domaines de recherche et d'enseignement on se retrouve souvent à réfléchir seuls » (E2).

La difficulté à travailler en groupe est, en partie, liée au manque de temps : "On est quasiment tous en sur-service [...], je n'ai plus assez de temps pour renouveler mon enseignement, me poser, retravailler dessus, proposer autre chose aux étudiants» (E2), mais aussi liée à une absence de soutien par les pairs. Également partagé par E3, celui-ci estime que les modifications qu'il a apporté à ses méthodes d'enseignement (en préférant commencer ses cours par des questionnements et des démonstrations pratiques avant le formalisme et les outils "pour donner envie de comprendre ", et en prenant davantage soin de ses supports) vont à contre-courant de l'avis de ses collègues : «les scientifiques pensent que c'est seulement le fond qui compte».

Selon l'enseignant E4, les étudiants vivent un décalage important. Il décrit des étudiants «intimidés » au début d'une conférence lorsqu'il leur a demandé d'exécuter des expériences de coin de table. "Ils était dubitatifs, [...] ils faisaient vraiment du bout des doigts ce que [l'enseignant E1] leur demandait de faire ». Pourtant, il a suffi d'une seule séance pour que ces étudiants s'impliquent et y trouvent visiblement de l'intérêt : «À la fin, ils proposaient eux-mêmes les réponses, les explications, ou ils essayaient d'autres choses, sans forcément de consignes ». La peur du jugement du professeur, connu pour dispenser des cours chargés d'équations complexes, a pu être à l'origine de cette gêne : "Je pense que si c'était nous [amàco] qui leur avions demandé, ils auraient eu beaucoup moins de mal [...] 
parce qu'ils ne [nous] connaissent pas. Là, il y a deux ans de passif, et à mon avis ça joue. En me mettant à leur place, je peux facilement imaginer ce genre de choses ".

Les entretiens avec les enseignants suggèrent que les habitudes des étudiants seraient plus facilement modifiables que celles des enseignants. Pour ces derniers, le changement demande soit plus de temps, soit plus d'investissement, ce qui reste difficile à trouver dans les deux cas, mais qui n'est pas impossible. L'enthousiasme reste un combustible puissant capable de percer les fissures et d'alimenter la flamme de la créativité et de l'innovation.

\section{Peut-on parler d'un effet amàco?}

88 Les données recueillies indiquent que la collaboration entre amàco et l'Insa Lyon a eu des effets. Les enseignants interrogés ont changé leurs pratiques pédagogiques introduisant plus d'expérimentation en classe et plus d'approches éco-responsables en lien avec les éco-matériaux; ils ont changé de posture vis-à-vis des étudiants en les accompagnant davantage et en encourageant plus l'expression de la créativité; ils ont augmenté leur intérêt pour le décloisonnement des départements et la prise en compte de la diversité des étudiants. Si les orientations et le degré des changements varient d'un enseignant à l'autre, les prises de conscience, elles, sont partagées et suggèrent qu'ils sont prêts à renouveler cette expérience, pour peu que le contexte ou la politique de l'établissement y soit favorable, notamment en termes de valorisation du travail effectué. De leur côté, les étudiants partagent leur optimisme à l'issue de leur expérience hors des sentiers battus. L'initiation à de nouvelles méthodes pédagogiques ne les a pas déstabilisés.

89 De façon générale, Micodu a été l'occasion pour les étudiants comme pour les enseignants de sortir du cadre habituel. L'approche inter- ou transdisciplinaire a été une vraie découverte pour tous. Plus encore, il a été un moyen de se projeter virtuellement hors des murs de l'institution et de pouvoir s'affranchir des règles internes et des mentalités sociales liées à l'apprentissage et à l'enseignement du métier d'ingénieur. Au-delà des apprentissages réalisés (travail en équipe, confrontation de points de vue, etc.), l'option transversale a fourni un terreau favorisant l'ouverture et la réceptivité à l'innovation. Pour les enseignants, pris par leur charge de travail et un sentiment de solitude, l'expérience hors-cadre pourrait avoir eu l'effet d'une soupape. Elle offre la possibilité de s'essayer à d'autres méthodes d'enseignement orientées vers les savoir-être et les savoir-faire, susceptibles d'apporter plus de satisfaction et sans doute de combler certaines frustrations du métier d'enseignant-chercheur.

90 À la lecture des données, l'efficacité d'un enseignement amàco et son originalité se situeraient surtout dans la capacité à toujours proposer un positionnement innovant par rapport aux méthodes et aux contenus habituellement enseignés. À la question de savoir si la terre est un moyen d'expérimenter ou un moyen de construire, la réponse à apporter serait de dire, dans le cas de Micodu, que c'est les deux à la fois.

91 Ce retour d'expérience de la collaboration d'amàco avec l'Insa Lyon pourrait paraitre difficile à transférer en raison de ses spécificités. Pourtant, le bilan révèle que la réussite de l'expérience ne dépend pas exclusivement du sujet de l'enseignement et qu'elle pourrait être reconduite avec succès avec d'autres thématiques. L'Insa Lyon partage, avec de nombreux autres établissements d'enseignement supérieur, le cloisonnement de ses filières, la surcharge de travail des enseignants, la priorité 
donnée à l'enseignement transmissif, mais aussi le fait qu'elle est, plus implicitement, le lieu d'appropriation des normes culturelles et professionnelles des métiers d'ingénieurs auxquels elle forme. C'est peut-être précisément parce que la pédagogie soutenue par amàco vient toucher à cet implicite, qu'elle permet d'envisager un changement en bousculant ce qui est perçu comme sclérosé et inadapté aux besoins sociétaux d'aujourd'hui.

Cet article examine la transposition des principes de la philosophie de la matière d'amàco à une ingénierie pédagogique qui met l'expérimentation directe avec la matière au cœur des pratiques pédagogiques. Notre démarche d'observation cherche à comprendre les effets de l'expérience sur l'expérience formative des étudiants et les changements dans les pratiques d'enseignement des enseignants. Si les effets formateurs sont encore difficiles à identifier clairement, les effets transformateurs des pratiques pédagogiques sont, en revanche, plus appréhensibles et très significatifs.

Force est de reconnaître que la mesure des effets d'une innovation pédagogique sur les apprentissages des étudiants reste une démarche délicate pour deux raisons. D'une part, il est impossible, voire irréaliste de vouloir établir un lien de causalité entre un contexte pédagogique et les apprentissages. D'autre part, si une telle relation devait exister, elle ne pourrait être mesurée que dans le temps, pour autant que l'on puisse isoler un seul facteur explicatif (ce qui semble peu probable vu la complexité des phénomènes sociaux et humains). Il nous faut, dès lors, nous tourner vers les forces invisibles en mouvement dans le paysage pédagogique. Les étudiants ayant suivi l'option Micodu témoignent de leur intérêt pour les enjeux de la construction durable et semble optimistes quant à leur capacité à contribuer aux changements dans le monde professionnel de la construction. Or, cette intentionnalité n'est pas réservée aux seuls étudiants issus de Micodu, elle semble s'étendre aux étudiants ingénieurs en général et trouver un écho dans l'idée de reconnaître l'importance à accorder à la formation d'« ingénieurs engagés ».

Du côté des forces visibles, nous trouvons les transformations observées chez les enseignants. Ici, la participation à une innovation pédagogique modifie de manière sensible les pratiques d'enseignement qui ne sont plus les mêmes. En même temps que le changement se fait, les résistances se font connaître et les enseignants se trouvent alors impliqués dans l'émergence d'une nouvelle culture pédagogique pour leur établissement. La naissance du groupe «ingénieur.e.s engagé.e.s » témoigne de cette volonté d'essaimer les bénéfices de l'expérimentation Micodu à toute l'offre de formation.

95 Notre expérience indique que les mouvements sont à l'œuvre et que les changements auront lieu à leur rythme. Cela invite à penser l'accompagnement au changement et, pour amàco, à réfléchir à la meilleure manière de poursuivre ses contributions dans le domaine de l'innovation et de la reconnexion à soi et au monde, en transformant les résistances en opportunités.

Remerciements : Les auteures de l'article souhaitent témoigner leur gratitude aux enseignants de l'Insa Lyon pour s'être sincèrement prêtés au jeu des entretiens, à l'équipe de formation amàco ayant participé à l'expérience, ainsi qu'à Léa Genis pour son regard extérieur sur l'analyse de données. 


\section{BIBLIOGRAPHIE}

Anger, R. (2011). Approche granulaire et colloïdale du matériau terre pour la construction. (Thèse de doctorat inédite). Institut national des sciences appliquées de Lyon, France.

Anger, R. et Fontaine, L. (2016). Matières animées. Le Philotope, 12, 225-234.

Astolfi, J.-P. (2015). L'erreur, un outil pour enseigner. Issy-les-Moulineaux, France : ESF éditeur.

Bisiaux, M. M., Fontaine, L., Anger, R. et Houben, H. (2015, juin). Enseigner la matière pour construire durable. Le projet pédagogique Atelier Matières à Construire. Communication présentée au colloque Question de pédagogie pour l'enseignement supérieur (QPES), Brest, France.

Bisiaux, M.M., Tric, Z., Houben, H., Fontaine, L., Anger, R. et Berthiaume, D. (2017, juin). Former à la matière pour se connecter à soi, à l'autre et au monde. Communication présentée au colloque Question de pédagogie pour l'enseignement supérieur (QPES), Grenoble.

Daele, A. et Berthiaume, D. (2013). Comment structurer les contenus d'un enseignement? Dans D. Berthiaume et $\mathrm{N}$. Rege Colet (dir.), La pédagogie de l'enseignement supérieur : repères théoriques et applications pratiques. Tome 1 : Enseigner au supérieur (p. 87-102). Berne, Suisse : Peter Lang.

Damasio, A.R. (1995). L'erreur de Descartes : la raison des émotions. Paris, France : Odile Jacob. Lemaître, D. (2015, juin). Pourquoi innover ? L'injonction pédagogique et ses enjeux éducatifs. Communication présentée au colloque Questions de pédagogie pour l'enseignement supérieur (QPES), Brest.

Fontaine, L., Moevius, M., Bisiaux, M. M., Neuville, J.-P. et Prud'homme, E. (2017, mars). Pédagogie alternative pour une ingénierie engagée. Communication présentée au colloque Pédagogie et Formation du groupe INSA, Lyon.

Mezirow, J. (1997). Transformative Learning: Theory to Practice. New Directions for Adult and Continuing Education, 74, 5-12.

Neuville, J.-P., Bouyer, M. et Lefèvre, F. (2017, mars). Quelle école pour élèves-ingénieurs 2.0? Communication présentée au colloque Pédagogie et Formation du groupe INSA, Lyon.

Rege Colet, N. (2016). From content-centred to learning-centred approaches : shifting educational development paradigm in higher education. Journal of Educational Administration and History, 49(1), 72-86.

Rege Colet, N. et Berthiaume, D. (2013). Comment choisir des méthodes d'évaluation adaptées? Dans D. Berthiaume et N. Rege Colet (dir.), La pédagogie de l'enseignement supérieur : repères théoriques et applications pratiques. Tome 1 : Enseigner au supérieur (p. 55-71). Berne, Suisse : Peter Lang.

\section{NOTES}

1. L'atelier matières à construire bénéficie de l'aide de l'État gérée par l'Agence Nationale de La Recherche au titre du programme Investissements d'Avenir des IDEFI ANR-11-IDFI-0008. 


\section{RÉSUMÉS}

Cet article rend compte d'une innovation pédagogique menée dans une école d'ingénieurs par une association indépendante : l'atelier matières à construire - amàco. Le projet visait à modifier les conceptions et les pratiques dans le domaine de la construction durable et le défi consistait à intégrer, dans l'ingénierie pédagogique, les principes d'une philosophie de la matière qui invite à faire corps avec la matière. Après trois années d'une expérimentation pédagogique avec la matière, dans le cadre d'un soutien financier à l'innovation, une démarche d'investigation, basée sur la théorie ancrée, a été entreprise pour observer, d'une part, les effets sur l'expérience formative des étudiants et le développement de leurs compétences professionnelles et, d'autre part, les modifications des pratiques d'enseignement des enseignants impliqués dans le projet. L'analyse montre que les changements les plus apparents concernent les enseignants qui transforment radicalement leur enseignement au-delà de l'expérience. Les effets sur le développement professionnel des étudiants sont plus difficiles à saisir bien qu'il y ait de bonnes indications qu'ils entrent dans le monde professionnel avec une vision accordant plus de place aux enjeux de la construction durable et au renouvellement du contact entre l'homme et son environnement.

This paper describes a pedagogical innovation carried out by amàco in an engineering school. The purpose of the project was to change conceptions and practices in the field of sustainable building and construction by permeating the instructional design with the core principles of an innovative philosophy based on direct contact with physical matter. After three years of experimenting with matter in the context of seed funding for innovation, a exploratory inquiry based on grounded theory was set up in order to observe the effects on the students' learning experience and professional development, and the changes in teaching practices for the teachers involved in the project. The results show that the clearest transformations concern the teaching practices that expand well beyond the project. The effects on professional development of students are more slippery to catch although there is good indications that they enter the work force with a vision that takes into account the issues of sustainable building and construction and pays attention to the need to reconnect mankind to his environment.

\section{INDEX}

Mots-clés : innovation pédagogique, philosophie de la matière, construction durable, théorie ancrée, pratiques pédagogiques innovantes, développement professionnel

\section{AUTEURS}

\section{MARION M. BISIAUX}

Atelier matières à construire amàco, c/o Les Grands Ateliers, Villefontaine, France.

\section{LAETITIA FONTAINE}

Atelier matières à construire amàco, c/o Les Grands Ateliers, Villefontaine, France. CRAterre AEetCC ENSAG UGA, Grenoble, France. 


\section{NICOLE REGE COLET}

Yggdrasil Living Wholeness, Gudhjem, Danemark. 